11-78 mg./24 hr.). Free (i.e. non-peptide) hydroxylysine is usually present only in trace amounts, except during a high collagen diet. An excess of free hydroxylysine was found in Case $1(10.0 \mathrm{mg} . / 24 \mathrm{hr}$.) and Case 2 $(12.3 \mathrm{mg} . / 24 \mathrm{hr}$.). Case 1 also excreted an excess of citrulline $(8.8 \mathrm{mg} . / 24 \mathrm{hr}$.).

Since no hydroxylysine was detected in the fasting bloods of controls or of patients, a renal origin of the aminoaciduria seemed more likely than a metabolic block. Results of hydroxylysine tolerance and renal clearance tests supported this view. Total urinary hydroxyproline was in the upper range of normal.

Since hydroxylysine and hydroxyproline in animal tissues are found almost exclusively in collagen, the ratio of these amino acids in urinary peptides might be expected to be constant provided that their rates of further metabolism were similar. This was investigated in 22 subjects on low collagen diets, 19 of whom were normal individuals, and 3 patients with Marfan's syndrome known to have high excretions of urinary total hydroxyproline. After hydrolysis of urinary peptides, a direct relation was found between the excretion of total hydroxylysine and hydroxyproline.

\section{Morphology and Pathology of Hip-Joint at Birth}

\section{Peter M. Dunn \\ Bristol Royal Hospital for Sick Children}

Examination at necropsy was made of 46 hip-joints belonging to 23 infants dying before or shortly after birth; 22 joints were normally formed, 1 was dysplastic without being dislocated, and the remaining 23 were either dislocated (16) or dislocatable (7). The status of all but one of the joints was correctly assessed by manipulative examination of the hip before dissection; the only error concerned the dysplastic hip which was wrongly thought to be a case of 'fixed' dislocation. The morphology of the normal hip-joint in the 2nd and 3rd trimesters of pregnancy and the spectrum of pathology shown by the dislocated hips were discussed. The visual and radiographic appearance of the hips dissected, normal, and abnormal, dislocated, and reduced, were illustrated.

\section{Neurological Assessment of Gestational Age in Newborn}

\section{Victor Dubowitz \\ University of Sheffield}

Various parameters recommended by previous authors have been assessed in about 150 newborn infants of varying gestational age and birthweight. A number of additional criteria for neurological assessment, thought to be of potential value in the assessment of neurological maturity, have also been included and compared with the standard methods.

The results have shown that it is impossible to draw a clear-cut correlation between gestational age and the presence or absence of a particular reflex or posture.
The range of variation may cover 4 to 6 weeks of gestation. This is in striking contrast to the apparent accuracy of the clinical estimate of gestational age based on these parameters. The clinical impression is probably based subconsciously on the sum total of the various criteria rather than on individual ones. A scoring system for all parameters and a total score for assessment of gestational age should prove more reliable than individual criteria or the subjective clinical impression. Nerve conduction velocity varies with gestational age and provides a useful objective parameter for comparison with clinical observations.

\section{Initial Experience with an Impedance Apnoea Monitor}

\section{H. M. Walker and Phyllis Robbie Department of Child Health,}

University of Dundee, and Dundee Royal Infirmary

With the ever-increasing shortage of nurses, the problem of providing adequate observation for the infant subject to apnoea has, in many units, already become serious. Recourse must be made, therefore, to instrumental monitoring, and the purpose of this paper is to report the early experience with the Airshields Impedance Apnoea Monitor.

An analysis of 708 alarms indicates that $600(85 \%)$ occurred before the apnoea was seen by the nursing staff. In $95(13 \%)$ there was a colour change noted at the time of the alarm. The nursing staff were not specifically asked to note the degree of cyanosis but it was recorded in 60 instances. In $49(52 \%)$ the colour change was mild and in $11(12 \%)$ it was moderate or severe.

Respiration was re-established spontaneously with automatic stopping of the alarm in $230(32.5 \%)$, but in $439(62 \%)$ the nurse had time to reach the infant and stimulate respiration before the next breath occurred. Some of these infants would doubtless have restarted breathing if left undisturbed for longer, but this figure indicates the frequency with which infants remain apnoeic for at least 20 or 30 seconds, the time lapse intervals before alarm which can be preselected by a control on the instrument. In the remaining $39(5.5 \%)$ this information was not recorded or was misrecorded.

False alarms, i.e. alarm signals when the infant was still breathing well, occurred in 47 infants $(7 \%)$; in 9 (1\%) this information was not recorded. In $652(92 \%)$ the alarms were regarded as a true indication of 20 or 30 seconds' apnoea, the time lapse interval used in most infants. In no case in this series did the alarm fail to indicate cessation of respiratory movements.

\section{Survey of Childhood Asthma in Aberdeen}

Blanche Dawson, Gordon Horobin, Raymond Illsley, and Ross Mitchell Department of Child Health, University of Aberdeen, and M.R.C. Medical Sociology Research Unit

The sample used was a 1 in 5 of all children attending Aberdeen primary schools in 1962 and still resident in 
Aberdeen in 1964-65 when interviewing was carried out. From this sample of 2500,300 possibly asthmatic children were medically examined and their parent or guardian interviewed by a paediatrician. 121 children were confirmed to be asthmatic. There were 84 boys and 37 girls: 16 of the 20 severe cases were boys. Age of onset of asthma showed that $98(80 \%)$ had experienced their first attack by the age of 5 .

There was a family history of asthma among first degree relatives in $64(53 \%)$, of eczema in $14(12 \%)$, and of allergic rhinitis in $21(17 \%)$. The asthmatic children themselves showed other allergic manifestations: 35 $(29 \%)$ had had infantile eczema, $27(22 \%)$ had had flexural eczema, and $49(37 \%)$ had had allergic rhinitis.

Review of therapy revealed that only one of the asthmatic children had received steroids before the survey.

The group of 121 were graded according to severitymild (50), moderate (51), and severe (20). Allocation was made on the basis of history and examination. Subsequent evaluation of pulmonary function tests showed good correlation with this grading.

Heights and weights of children in all grades tended to be below the mean, but those in the severe grade were at or less than $2 \mathrm{SD}$ below the mean.

Sociological data obtained and evaluated revealed that there were significantly more of the severe asthmatics in the semi- and unskilled manual classes. The severe asthmatics tended to occur in families of 4 or more, regardless of social class. The IQ score at $7+$ years showed that all asthmatics tended to score higher than the rest of the population. This was particularly apparent in the semi- and unskilled manual classes where the mean IQ of the asthmatics was 109 and that of the population 102 .

The over-all prevalence of asthma in this sample of Aberdeen schoolchildren was $4 \cdot 8 \%$.

\section{Arterial Blood Gas Tensions and $p H$ in Acute Asthma in Childhood}

\author{
H. Simpson \\ Department of Child Life and Health, \\ University of Edinburgh
}

The arterial blood gas tensions and $p \mathrm{H}$ in 21 children studied during 24 acute attacks of asthma were reported. All were hypoxic on admission to hospital, and in 10 there was evidence of $\mathrm{Co}_{2}$ retention. Cyanosis, invariably present when the arterial oxygen saturation was below $85 \%$, and restlessness in patients breathing air, were the most reliable indices of the severity of hypoxia. There were no reliable clinical guides to the $\mathrm{PCO}_{2}$ level. Conventional oxygen therapy in tents (25-40\%) did not always relieve hypoxia, and in 3 cases the administration of oxygen at a concentration of $40 \%$ or over failed to produce a normal arterial oxygen tension. Uncontrolled oxygen therapy could aggravate respiratory acidosis, and 2 children developed $\mathrm{CO}_{2}$ narcosis while breathing oxygen. The necessity for blood gas measurements in the management of severe acute asthma in childhood was emphasized.

\section{Some Biochemical Aspects of Intrauterine Growth Retardation}

\author{
Forrester Cockburn \\ Department of Child Life and Health, \\ University of Edinburgh
}

A group of 9 infants with birthweight below the 10th centile for gestational age, born to mothers with preeclamptic toxaemia and a low urinary excretion of oestriol, were studied at birth and daily during the first week of life.

Samples of blood were obtained at caesarean section from maternal vein, umbilical vein, and umbilical artery. At 24-hour intervals capillary blood samples were obtained 2-2 $\frac{1}{2}$ hours after standard evaporated milk feeds. Values obtained from a group of 15 normally grown infants delivered by caesarean section were used as controls.

Haematocrit and $p \mathrm{H}$ values in maternal and fetal samples of control and low birthweight (LBW) groups were comparable at birth. During the first 4 days after birth the haematocrit was significantly higher in the LBW infants. Capillary $p \mathrm{H}$ values were lower in the LBW group throughout the first week. A mean maternal vein-umbilical vein difference of $24 \mathrm{mg}$. glucose $/ 100 \mathrm{ml}$. plasma was found in the LBW group compared with $10 \mathrm{mg} . / 100 \mathrm{ml}$. plasma in the normal. The umbilical venous and arterial glucose concentrations in the LBW infants were significantly below those of the controls and remained lower throughout the first week. Total plasma protein concentrations of the LBW group were significantly below normal in maternal as well as in all the perinatal samples. There were no gross differences in umbilical vein plasma amino acid concentrations apart from a higher taurine concentration in the LBW. samples. The over-all ratio of umbilical vein/maternal vein plasma amino acid concentration was reduced from 1.9 (control) to 1.5 (LBW) due to an increase in the toxaemic mothers' amino acid concentrations towards the non-pregnant values.

Plasma calcium was significantly lower and inorganic phosphorus higher in the umbilical vein plasma of the LBW infants. Magnesium values were comparable throughout. Concentrations of copper were higher in the plasma of the toxaemic mothers but fetal concentrations were normal.

The relevance of these findings in relation to placental function and the subsequent development of the infants was discussed.

\section{Folic Acid Trends and Prophylaxis in Prevention of Megaloblastic Anaemia of Infants of Low Birthweight}

T. F. P. Mackintosh, M. K. Strelling,

C. H. M. Walker, and H. B. Goodall

Departments of Child Health and Pathology,

University of Dundee, and Dundee Royal Infirmary

In the first phase of this study the trends in whole 